\title{
Multilinguales
}

\section{La morphologie nominale et les segments flottants en kabyle}

Nominal morphology and floating segments in kabyle

\section{Samir Ben Si Said}

\section{(2) OpenEdition}

\section{Journals}

\section{Édition électronique}

URL : https://journals.openedition.org/multilinguales/5353

DOI : $10.4000 /$ multilinguales.5353

ISSN : 2335-1853

\section{Éditeur}

Université Abderrahmane Mira - Bejaia

\section{Référence électronique}

Samir Ben Si Said, "La morphologie nominale et les segments flottants en kabyle », Multilinguales [En ligne], 13 | 2020, mis en ligne le 15 juillet 2020, consulté le 18 mai 2021. URL : http:// journals.openedition.org/multilinguales/5353; DOI : https://doi.org/10.4000/multilinguales.5353

Ce document a été généré automatiquement le 18 mai 2021

\section{cc) $(1) \ominus$}

Multilinguales est mise à disposition selon les termes de la Licence Creative Commons Attribution Pas d'Utilisation Commerciale - Pas de Modification 4.0 International 


\title{
La morphologie nominale et les segments flottants en kabyle
}

\author{
Nominal morphology and floating segments in kabyle
}

\author{
Samir Ben Si Said
}

1 Les objets phonologiques peuvent être présents dans la racine lexicale mais absents de la réalisation de surface, comme l'ont montré les études phonologiques, dès les années 80. En effet, il a été démontré que des consonnes flottantes peuvent exister au niveau sous-jacent comme le montre Encrevé (1988) pour le phénomène de liaison en français, ainsi que des voyelles flottantes dans le comportement des suffixes de voyelle initiale tchèque _ov ou_ity (Ziková et Scheer 2009). Il a également été avancé que certains tons peuvent se comporter de la même manière et donc être caractérisés comme des objets flottants (Hyman 2007, Paster 2003, Zimmermann 2017 parmi d'autres). Le fait que de tels objets puissent flotter est dû à la configuration restreinte de l'espace syllabique. En conséquence, nous pouvons dire que la structure peut empêcher la prononciation du matériel mélodique. C'est-à-dire que la structure n'offre pas assez d'espace à tous les objets mélodiques (consonnes et voyelles) pour être associés et réalisés en surface.

2 Je soutiendrai ici que le pluriel externe (formé par suffixation) en kabyle peut s'expliquer par le même schéma. Je propose que, malgré l'apparente irrégularité, la formation du pluriel en kabyle est parfaitement régulière. Je montre en effet que la formation du pluriel est cohérente et simple dès lors que l'on fait l'hypothèse gabaritique et que l'on adopte l'hypothèse de la longueur virtuelle (Lowenstamm 1991, Bendjaballah 2005). Dans ce cadre, il n'y a en réalité qu'un seul suffixe du pluriel, -n. C'est alors que les processus supplémentaires qui interviennent lors du passage du singulier au pluriel découlent simplement des contraintes imposées par le gabarit. Dans ce contexte, je montrerai que le pluriel est uniforme et il a un gabarit de 5 unités CV et les éléments mélodiques correspondant au pluriel appartiennent bien à l'entrée lexicale mais, au singulier, ils demeurent flottants ou associés à une seule position si le gabarit est trop petit (inférieur à $5 \mathrm{CV}$ ). La conséquence en est que leur interprétation phonétique est altérée : les segments flottants ne sont pas prononcés, et les voyelles associées à une seule position apparaissent à la surface en tant que schwa ou zéro. C'est 
au moyen d'une analyse de ces éléments mélodiques en tant que segments flottants (ou simplement associés) appartenant à la racine que l'on peut dépasser l'allomorphie à laquelle les analyses traditionnelles étaient contraintes.

\section{Quelques caractéristiques du pluriel externe}

Dans cette partie, je présente l'analyse classique de la formation du pluriel externe en kabyle.

\section{Le pluriel en kabyle}

4 Il existe trois types de pluriels en Kabyle ${ }^{1}$ le pluriel externe, appelé "pluriel en -n",

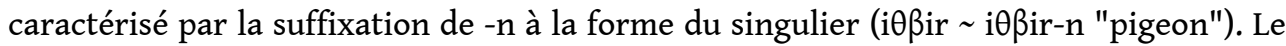

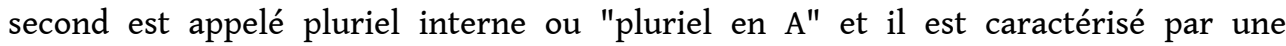
modification de la qualité des voyelles de la racine, ainsi que par l'apparition d'un A en dernière position vocalique (aүanim iүunam "roseau"). Et le troisième est appelé pluriel mixte qui combine les marques des deux premiers, c'est-à-dire, il est caractérisé par la suffixation $d u-n$ du pluriel externe ainsi que la marque du pluriel interne qui est la présence de la voyelle A en dernière position vocalique (azal izilan "clarté du jour"). .

Il est à noter que l'alternance de la voyelle initiale entre le singulier et le pluriel se produit dans $97 \%$ des noms (Basset 1945). Diachroniquement, il s'agit d'un article préfixé qui ne peut cependant pas être dissocié de la racine (Brugnatelli 1987, 1997, Stumme 1899 et Vycichl 1957). Cette alternance ne joue aucun rôle dans la démonstration ci-dessous.

\section{L'analyse classique du pluriel externe et ses problèmes}

5 En plus de la suffixation d'un -n, le pluriel externe peut montrer en surface ce qui est traditionnellement interprété comme une allomorphie du suffixe (Chaker 1983). Cette variation est illustrée sous (1) où la seule propriété stable du suffixe semble être la présence d'un $-n$.

(1)

\begin{tabular}{|c|c|c|c|}
\hline singulier & pluriel & suffixe & glose \\
\hline $\mathrm{i}-\theta \beta \mathbf{i r}^{2}$ & i- $\theta \beta$ ir-n & $-n$ & pigeon \\
\hline a- $\beta ә q q a$ & $\begin{array}{l}\text { i- } \beta ə q q a- \\
\text { jən }\end{array}$ & -jən & gifle \\
\hline a-jawa & $\begin{array}{l}\text { i-jawa- } \\
\text { wən }\end{array}$ & -wən & $\begin{array}{l}\text { Kabyle habitant la région montagneuse au Nord de la chaîne du } \\
\text { Djurdjura }\end{array}$ \\
\hline alma & alma-Өən & 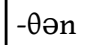 & prairie \\
\hline izəm & izm-awən & -awən & lion \\
\hline
\end{tabular}




\begin{tabular}{|l|l|l|l|}
\hline ifər & afr-iwən & -iwən & aile \\
\hline
\end{tabular}

On voit que les allomorphes du suffixe nombreux et variés, et la seule propriété stable est la présence d'un -n. Dans l'analyse traditionnelle (Chaker 1983, Mammeri 1986 parmi d'autres), la segmentation du suffixe se fait par rapport à la forme du singulier : tous et seulement les segments qui sont présents au pluriel mais absents au singulier appartiennent au suffixe.

6 En dehors de l'apparente irrégularité du suffixe, il y a aussi des voyelles qui sont absentes au singulier mais qui apparaissent au pluriel.

(2)

\begin{tabular}{|c|c|c|c|}
\hline voyelle absente au sg & singulier & pluriel & glose \\
\hline \multirow[t]{3}{*}{ a } & uffən & uffan-n & chacal \\
\hline & ið̃mər & iðmar-n & poitrine \\
\hline & izimər & izamar-n & mouton \\
\hline \multirow[t]{3}{*}{ i } & if $\int \partial w$ & adfiw-n & corne \\
\hline & it $t^{\tau} t^{\top} \partial w$ & $a t^{\top} t^{5} i w-n$ & œil (augmentatif) \\
\hline & ifər & afriw-n & aile \\
\hline
\end{tabular}

Par ailleurs, les éléments qui apparaissent au pluriel mais absents au singulier ne peuvent être prédits : ils doivent faire partie intégrante de la représentation lexicale de la racine. Pour des raisons qui seront développées plus loin, ils n'apparaissent pas au singulier.

\section{Ramener l'irrégularité à la régularité}

7 On constate donc une formation irrégulière du pluriel : les locuteurs doivent apprendre par cœur quel allomorphe du pluriel et quelles modifications internes surviennent avec quelle racine. Je propose que malgré son apparente irrégularité, la formation du pluriel est parfaitement régulière. Ainsi, moyennant une analyse en termes de gabarits et quelques outils empruntés à la phonologie de gouvernement, je montrerai que la formation du pluriel est cohérente : il n'y a en réalité qu'un seul suffixe du pluriel -n, et les différents processus qui interviennent lors du passage du singulier au pluriel sont nécessaires étant donné les contraintes imposées par le gabarit du pluriel.

Par ailleurs, si on considère que la seule marque concaténative du pluriel que l'on peut déterminer est $-\mathrm{n}$, il faut alors statuer sur les segments qui sont présents au pluriel mais absents au singulier. Ils sont identifiés sous (3). 
(3)

\begin{tabular}{|c|c|c|c|c|}
\hline singulier & pluriel & $\begin{array}{l}\text { élément présent } \\
\text { au pl. }\end{array}$ & suffixe & glose \\
\hline $\mathrm{i}-\theta \beta \mathrm{ir}$ & i- $\theta \beta$ ir-n & - & $-n$ & pigeon \\
\hline a-ßәqqa & $\begin{array}{l}\mathrm{i}-\beta \text { } \beta q q a-j- \\
\mathrm{n}\end{array}$ & $\mathrm{j}$ & $-n$ & gifle \\
\hline a-jawa & $\begin{array}{l}\text { i-jawa-w- } \\
\text { n }\end{array}$ & W & $-n$ & $\begin{array}{l}\text { Kabyle habitant la région montagneuse au Nord } \\
\text { de la chaîne du Djurdjura }\end{array}$ \\
\hline alma & alma- $\theta-n$ & $\theta$ & $-n$ & prairie \\
\hline izəm & izm-aw-n & aw & $-n$ & lion \\
\hline ifər & afr-iw-n & iw & $-n$ & aile \\
\hline
\end{tabular}

Comme il a été soulevé dans Chaker (1983 : 90), il y a deux options analytiques : ou bien les segments qui apparaissent seulement au pluriel sont épenthétiques, ou alors ils appartiennent à la racine. Dans ce dernier cas, il faudra comprendre pourquoi ils sont absents au singulier. Je montrerai que la dernière option est la bonne : les segments en question font partie de la racine mais, à cause de la taille du gabarit du singulier qui ne leur offre pas assez d'espace pour s'associer, ils demeurent flottants.

\section{Argument contre l'option épenthétique}

8 L'argument contre la perspective épenthétique qui saute aux yeux est l'imprédictibilité des éléments qui apparaissent au pluriel. Des noms appartenant au même gabarit singulier de surface montrent des éléments mélodiques différents au pluriel.

(4)

\begin{tabular}{|c|c|c|c|c|c|}
\hline & gabarit sg. & singulier & pluriel & épenthèse & glose \\
\hline \multirow[t]{3}{*}{ a. } & $\mathrm{CCCV}$ & a-məçsa & i-məçsaw-n & w & berger \\
\hline & & а-вәгðа & i-вәrðaj-n & j & souris \\
\hline & & a-jəfki & i-jəfki $\underline{\theta}-n$ & $\theta$ & lait \\
\hline \multirow[t]{2}{*}{ b. } & $\mathrm{Vccc}$ & 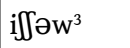 & afSiw-n & $\mathrm{i}$ & corne \\
\hline & & iffər & affarr-n & $a$ & ongle \\
\hline \multirow[t]{3}{*}{ c. } & $\mathrm{VCC}$ & isəm & ismaw-n & aw & nom \\
\hline & & ixəf & ixfaw-n & aw & extrémité \\
\hline & & івәð & івівð-n & ів & cendre \\
\hline
\end{tabular}


Si on veut éviter de multiples allomorphes au pluriel, l'imprédictibilité des segments présents au pluriel mais absents au singulier n'autorise qu'une seule analyse: ces segments font partie de la racine.

\section{Le gabarit du pluriel et le flottement au singulier}

La généralisation que je présenterai dans cette section permettra d'unifier tous les pluriels et de montrer le flottement au singulier. Or cette généralisation, dont le cœur est un gabarit de 5 unités CV, n'est valable qu'en adoptant l'hypothèse de la longueur virtuelle des voyelles périphériques en kabyle (Bendjaballah 2005). Avant de présenter mon analyse du pluriel, j'introduis d'abord cette hypothèse, la notion de gabarit ainsi que le modèle $\mathrm{CVCV}$, trois outils théoriques nécessaires pour mon analyse.

\section{Le cadre CVCV}

La plupart des modèles autosegmentaux (Leben 1973, Goldsmith 1976 et McCarthy 1979) distinguent le niveau squelettal, qui détermine le nombre de segments d'une séquence, du niveau syllabique, qui précise leurs relations hiérarchiques au moyen d'une arborescence. Le cadre standard de la Phonologie de Gouvernement (Kaye, Lowenstamm \& Vergnaud 1985, 1990, Kaye 1990) n'y fait pas exception. Dans ce modèle, le niveau syllabique comporte les constituants attaque, rime et noyau, qui peuvent être simples ou branchants. Sont absents de l'inventaire la syllabe et la coda, qui n'existent pas en tant que tels.

11 L'approche CVCV (Lowenstamm 1996, Scheer 2004) se démarque en ce qu'elle attribue les effets syllabiques aux seules forces latérales. Dans ce cadre ne sont distingués que deux types de constituants : les attaques et les noyaux non branchants, qui se succèdent en alternance stricte, et qui figurent dans les représentations sous la forme de $\mathrm{C}$ et de $\mathrm{V}$ pour, respectivement, les attaques et les noyaux. Les consonnes ne sont pas spécifiées en termes d'attaque ou de coda, ce sont les relations qu'elles entretiennent entre elles ou avec les voyelles qui déterminent la structure syllabique d'une chaîne linéaire ${ }^{4}$.

Dans le modèle CVCV, une consonne finale (5a), une syllabe fermée ( $5 b)$, une attaque branchante $(5 \mathrm{c})^{5}$, une géminée $(5 \mathrm{~d})$ et une voyelle longue (5e) seront représentées de la manière suivante :

(5)

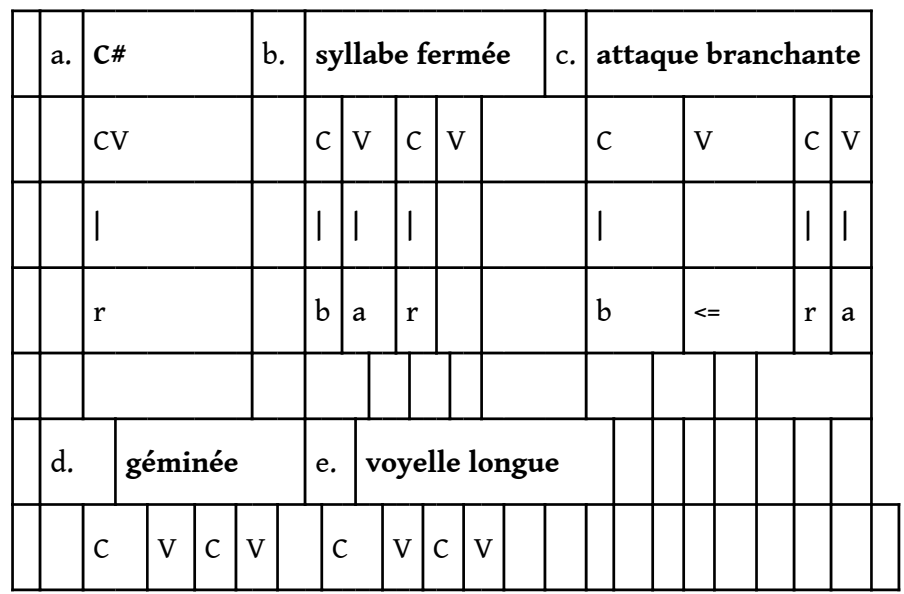




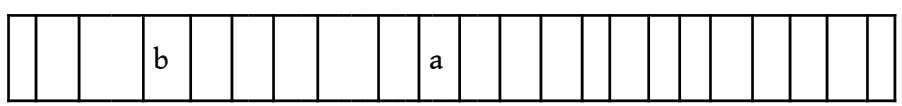

\section{Le gabarit}

Le gabarit, tel qu'il est conçu en afro-asiatique, est une séquence particulière de positions vocaliques et/ou consonantiques associée à une valeur morpho-sémantique (McCarthy 1979, 1982, 1983). Le gabarit a une taille fixe et doit être satisfait, i.e. rempli, quel que soit le matériel vocalique et consonantique de départ: ce principe a été formalisé par McCarthy (1979, 1981), McCarthy et Prince (1990) sous le nom de « Template Satisfaction Principle ».

En arabe classique, les différentes formes verbales et nominales obéissent à des gabarits particuliers, appelés traditionnellement binyanim (singulier de binyan). Il y a 10 formes verbales en arabe classique auxquelles on peut ajouter 5 autres formes qui sont rares et non productives. Chaque forme est associée à un certain sémantisme. Le tableau sous (6) récapitule les dix formes courantes.

(6)

\begin{tabular}{|c|c|c|}
\hline forme I & faYal & forme simple du perfectif actif \\
\hline forme II & faSYal & causatif \\
\hline forme III & faaSal & réciproque \\
\hline forme IV & Pa-fYal & causatif, estimatif \\
\hline forme $\mathrm{V}$ & ta-faYSal & réflexif causatif \\
\hline forme VI & ta-faaYal & réflexif réciproque \\
\hline forme VII & n-faYal & réflexif passif? \\
\hline forme VIII & iftaSal & réflexif \\
\hline forme IX & fYall & devenir + adjectif \\
\hline forme X & sta-ffal & réflexif de la forme IV \\
\hline
\end{tabular}

Ces formes ont une caractéristique essentielle: elles prévoient un nombre fixe de positions consonantiques. Si la racine a moins de consonnes qu'il n'est prévu par le gabarit, une des consonnes doit se propager ou une nouvelle consonne doit être insérée. Si la racine est trop grande, une ou des consonnes doivent tomber.

Pour illustrer le principe de satisfaction gabaritique, prenons l'exemple d'une forme de pluriel en arabe classique, sous (7), dont le gabarit comporte 4 positions consonantiques (McCarthy 1979). 
(7)

\begin{tabular}{|l|l|l|l|l|}
\hline & racine & singulier & pluriel : CaCaaCiC & glose \\
\hline a. & fndzn & findzaan & fanaadzin & «tasse » \\
\hline & srwl & sirwaal & saraawil & «pantalon » \\
\hline b. & Pmr & Pamr & Pawaamir & « ordre » \\
\hline & Hṛ & Har & PaHaarir & « chaleur » \\
\hline c. & sfrzl & safarzal & safaari3 & « coing » \\
\hline & Snkbt & Sankabuut & Sanaakib & " araignée » \\
\hline
\end{tabular}

Pour satisfaire le gabarit, quand la racine est trop petite sous (7b) des éléments mélodiques sont insérés ( $w$ dans Pawaamir) ou propagés ( $r$ dans PaHaariri). En revanche, lorsque la racine est trop grande, comme sous $(7 \mathrm{c})$, des éléments mélodiques tombent ( $l$ dans safarzal).

Le berbère, comme les autres langues afro-asiatiques, connaît les gabarits (Bendjaballah 1995, 1999, 2001, 2011 et passim, Ben Si Said 2014, Idrissi 2000, Lahrouchi 2001, 2003, 2009, 2013 et passim), même si leur existence et étendue sont sujettes à discussion.

En kabyle, dans le domaine nominal, on constate une activité gabaritique, notamment dans la formation du pluriel (Bendjaballah 2011, Bendjaballah et Haiden 2008, 2013 et Ben Si Saïd 2010, 2011, 2014). Voici quelques pluriels internes et pluriels mixtes en kabyle :

(8)

\begin{tabular}{|c|c|c|c|c|}
\hline & \multicolumn{2}{|l|}{ singulier } & \multirow{2}{*}{$\begin{array}{l}\text { pluriel } \\
\text { iCəCCan }\end{array}$} & \multirow{2}{*}{ glose } \\
\hline a. & & & & \\
\hline & \multirow{2}{*}{ VCCVC } & aßrið & ißərðan & « chemin » \\
\hline & & afraj & ifərjan & " clôture » \\
\hline & \multirow{2}{*}{ VCCC } & aðfəl & ¿ðəflan & « neige » \\
\hline & & ifдəə & ifəðnan & « gros orteil » \\
\hline & \multirow{2}{*}{ VCCiCiVc } & aðəbbuz & iðəbzan & «bâton » \\
\hline & & $a \int \partial b b a c ̧$ & ifəßçan & " grille» \\
\hline \multirow[t]{2}{*}{ b. } & & & iCuCaC & \\
\hline & & атадав & ітидав & «ronce» \\
\hline
\end{tabular}




\begin{tabular}{|c|c|c|c|}
\hline & ajazid $^{\complement}$ & ijuzad ${ }^{\varsigma}$ & «coq » \\
\hline \multirow{2}{*}{ VCVCC } & azarəz & izuraz & « corde » \\
\hline & ініqәl & iнuqal & " perdrix mâle» \\
\hline \multirow{2}{*}{ VCCVC } & annar & inurar & « aire à battre » \\
\hline & amkan & imuçan & « endroit» \\
\hline
\end{tabular}

On observe que quelle que soit la forme du singulier, les noms se neutralisent sous un seul gabarit du pluriel : iCaCCan sous (8a) et iCuCaC sous (8b).

\section{La longueur virtuelle des voyelles et l'interface phonétique- phonologie}

Dans une conception modulaire qui est celle de la grammaire générative (Fodor 1983), la phonétique et la phonologie constituent deux systèmes computationnels indépendants dont le premier travaille sur des objets articulatoires et/ou auditifs; et le second traite de représentations cognitives abstraites ${ }^{6}$.

L'objet de l'interface phonétique-phonologie est d'expliquer comment les deux modules communiquent (Kingston 2007, Woohyeok 2012). Comment des unités cognitives qui existent seulement dans le cerveau sont-elles transformées en des objets physiques et vice-versa?

On sait depuis longtemps que la relation entre les deux modules n'est pas toujours un à un : il peut y avoir une distorsion entre les représentations phonologiques et leurs interprétations phonétiques. Ainsi un objet phonologique peut avoir plusieurs interprétations phonétiques et vice-versa (Boersma 1998, Hamann 2011 et Scheer 2014, entre autres).

Un exemple tiré de Hamann (2011) est celui de la voyelle [e] en langue chukchi (parlée dans la région de Chukotka en Sibérie). Cette voyelle a une seule réalisation phonétique mais montre deux comportements phonologiques différents: /e1/ s'abaisse lors de l'harmonie vocalique, alors que /e2/ la déclenche. Un autre exemple donné par Scheer (2014) est celui de la voyelle $e$ en polonais. Cette voyelle montre deux comportements distincts: il y a des cas où $e$ palatalise (p. ex. lot - leci-e «vol (avion) au cas nominatif »), alors que dans d'autres cas il ne produit pas cet effet (lot - lot-em " vol (avion) au cas instrumental »). Les travaux sur le polonais en concluent que les deux $e$ sont deux objets phonologiques différents mais qui sont phonétiquement identiques.

Cette distorsion entre la phonétique et la phonologie a été développée en phonologie de gouvernement sous l'appellation interprétation phonétique (Harris et Lindsey 1995, Harris 1996). Dans cette optique, l'interprétation phonétique d'un objet phonologique est indépendante de celui-ci : elle a lieu après la fin de la computation phonologique et tout comme lors de la transformation de la structure morpho-syntaxique en objets phonologiques traduit, par une épellation (spell-out), le vocabulaire d'un module (phonologique) dans celui d'un autre module (phonétique).

En résumé, l'interprétation phonétique d'objets phonologiques peut être de trois types : 1) relation fidèle ( 1 à 1 ) où un objet phonologique est interprété tel quel par la 
phonétique (sous (9). 2) distorsion sous (9), i.e. un objet phonologique n'arrive pas en tant que tel en phonétique (p. ex. un objet long en phonologie qui arrive bref en phonétique et vice-versa). Et enfin 3) fusion (sous (9c), lorsque deux objets phonologiques sont interprétés comme un seul par la phonétique.

(9)

\begin{tabular}{|l|l|l|l|l|l|}
\hline & a. relation fidèle & \multicolumn{2}{|l|}{ b. distorsion } & \multicolumn{2}{|l|}{ c. fusion } \\
\hline représentation phonologique & /a/ & /aa/ & $/ \mathrm{r} /$ & $/ \mathrm{e} 1 /$ & $/ \mathrm{e} 2 /$ \\
\hline & & & & & \\
\hline interprétation phonétique & {$[\mathrm{a}]$} & {$[\mathrm{a}]$} & {$[\mathrm{b} / \mathrm{x}]$} & {$[\mathrm{e}]$} \\
\hline
\end{tabular}

La longueur virtuelle que j'introduis sous (11) illustre le cas sous (9), un seul par la phonétique. (9), une distorsion entre l'objet phonologique et sa valeur phonétique.

Dans l'analyse présentée dans cet article, j'adhère à la longueur dite virtuelle des voyelles en Kabyle: les voyelles périphériques (ou pleines) sont phonologiquement longues. Il s'agit d'une longueur phonologique qui n'est pas réalisée phonétiquement en tant que durée. La longueur en phonologie est bien toujours marquée en surface mais, d'une langue à une autre, la phonétique utilise des moyens différents pour l'exprimer. On dit qu'une voyelle est virtuellement longue lorsque sa longueur phonologique se manifeste autrement que par la durée en phonétique (Bucci 2013, Hammond 1997, Lowenstamm 1991, parmi d'autres).

Si on adapte le schéma sous (9b) pour les voyelles pleines du kabyle, on aura les représentations suivantes:

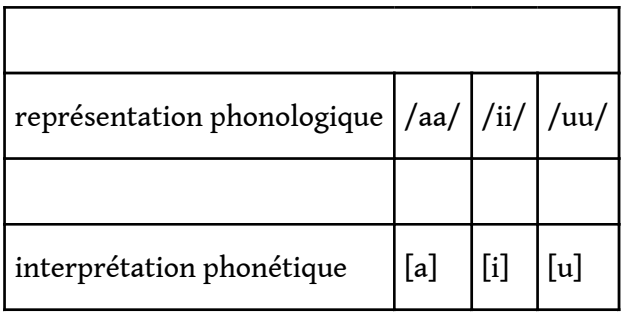

En revanche, une voyelle phonologiquement brève, c'est-à-dire associée à une seule position structurelle, apparait en surface (en phonétique) avec un timbre schwa (Bendjaballah 2005). Dans le cadre CVCV et selon l'hypothèse de la longueur virtuelle, les voyelles pleines du kabyle ont les représentations sous (11): les éléments A, I et U sont associées à deux positions vocaliques.

Lorsqu'une voyelle pleine est associée à une seule position vocalique, elle est réalisée schwa ou zéro selon les contraintes phonotactiques exigées par la langue (Bendjaballah $2005: 54)$. 
(11)

\begin{tabular}{|c|c|c|c|c|c|c|c|c|c|c|c|c|}
\hline C & $\mathrm{V}$ & C & V & C & V & C & V & C & V & C & V & $\mathrm{CV}$ \\
\hline & & A & & & & I & & & & U & & A/I/U \\
\hline & & [a] & & & & [i] & & & & {$[\mathrm{u}]$} & & [ə/ø] \\
\hline
\end{tabular}

J'ajoute que les éléments I et U sont épelés $j$ et $w$, respectivement, lorsqu'ils sont associés à une position consonantique (Kaye et Lowenstamm 1984). Les représentations de I/U associées aux deux types de constituants syllabiques sont données sous (12).

(12)

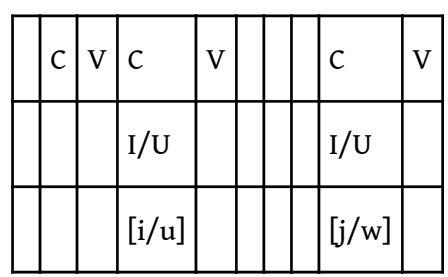

\section{La formation des noms en kabyle}

21 Je montrerai que le pluriel se ramène à un gabarit uniforme fait de $5 \mathrm{CV}$, sous l'hypothèse de la longueur virtuelle. Ce résultat acquis, je démontrerai qu'il repose crucialement sur la longueur virtuelle. Le fait que l'unification des pluriels ne puisse être acquise sans elle constitue alors un argument fort en sa faveur. Par ailleurs, je montre que le singulier a des gabarits différents et les segments mélodiques qui apparaissent au pluriel mais sont absents au singulier sont flottants à cause de la restriction de l'espace syllabique offert par le gabarit du singulier.

\section{Pourquoi la longueur virtuelle?}

La généralisation, selon laquelle le pluriel est régulier et impose une taille invariable de 5 unités $\mathrm{CV}$, est inattendue quand on regarde d'une part la variation que semblent présenter les faits de surface, d'autre part les traitements précédents qui ont tous conclu à l'irrégularité. Dans cette section, je montre que la régularité dégagée repose crucialement sur l'hypothèse de la longueur des voyelles périphériques (Bendjaballah 2005). Comme il est montré sous (13), sans cette hypothèse, aucune taille invariable au pluriel ne peut être dégagée.

(13)

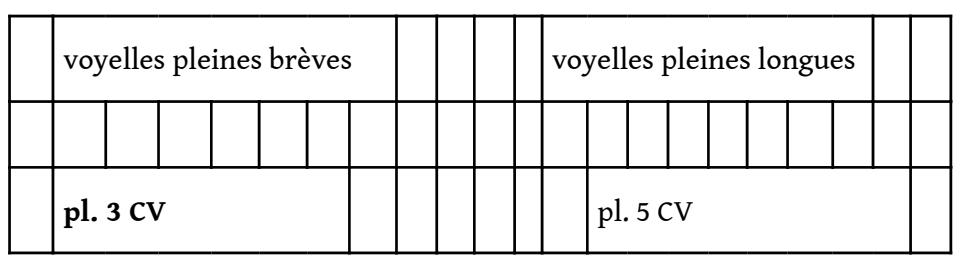




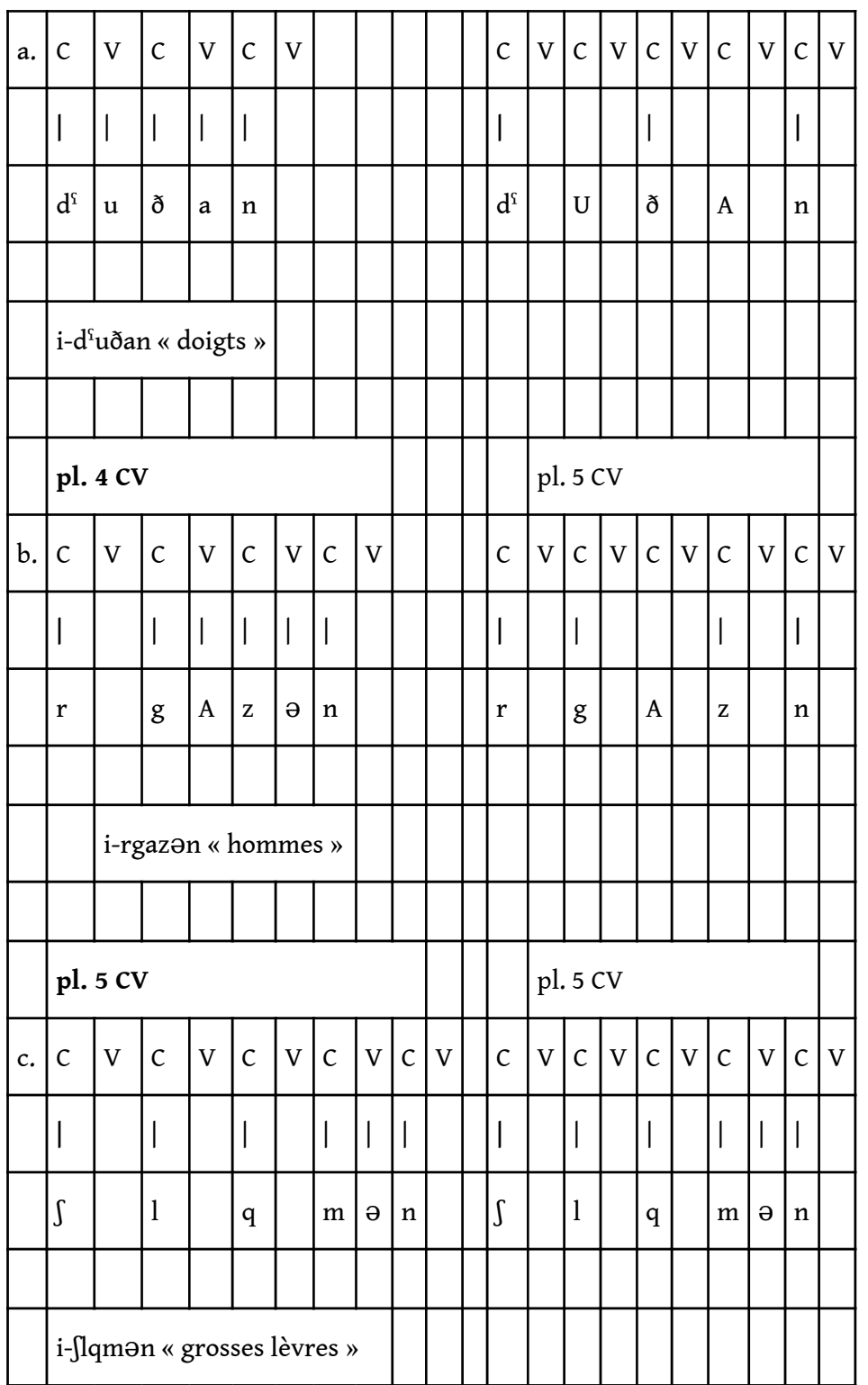

On voit que, sans la longueur virtuelle, la taille constante du pluriel ne peut être décrite : les pluriels font tantôt 3 , tantôt 4 et tantôt 5 unités $\mathrm{CV}$. Je conclus que seule l'hypothèse de la longueur virtuelle des voyelles pleines permet de découvrir la régularité que renferment les pluriels du kabyle.

\section{Dérivation du pluriel externe et du singulier}

Bien que la généralisation de la taille unique $(5 \mathrm{CV})$ soit valable pour tous les types de pluriel, je limite la démonstration au pluriel externe. Considérons à présent 3 pluriels externes de formes différentes: CCCVC-n, VCCVC-n et CVCV-n ${ }^{7}$ avec les singuliers associés.

(14)

\begin{tabular}{|l|l|l|l|l|}
\hline & gabarit & pluriel & singulier & glose \\
\hline
\end{tabular}




\begin{tabular}{|c|c|c|c|c|}
\hline a. & ccCVC-n & i-Səbbuß-n & a-Səbbu $\beta$ & chevelure \\
\hline & & i-fəqqiq-n & a-jəqqiq & fente \\
\hline & & i-qəswað-n & a-qəswað & enjambée \\
\hline & & i-məqlij-n & i-məqli & poêle \\
\hline & & $i-m t^{\mathrm{I}} t^{\mathrm{I}} a w-n$ & $i-m \partial t^{\mathrm{S}} t^{\mathrm{S}} \mathrm{i}$ & larme \\
\hline & & i-səqqi $\theta-n$ & a-səqqi & bouillons de couscous \\
\hline b. & VCCVC-n & anz $z^{\mathrm{f}} \mathrm{d}-\mathrm{n}$ & anz $z^{\mathrm{q}}$ əð & poil \\
\hline & & irri3-n & irri3 & braise \\
\hline & & affiw-n & iffəw & corne \\
\hline & & urgal-n & urgəl & figue précoce \\
\hline & & izmaw-n & izəm & lion \\
\hline & & uðmaw-n & uðəm & visage \\
\hline c. & CVCVC-n & i-zulis-n & а-zulis & boue \\
\hline & & i-waziw-n & a-waziw & volontaire \\
\hline & & i-nasur ${ }^{\mathrm{i}}-\mathrm{n}$ & a-nabur ${ }^{\mathrm{I}}$ & canicule \\
\hline & & i-Hulij-n & a-Huli & bouc \\
\hline & & i-juðuj-n & a-juðu & dépôt de détritus \\
\hline
\end{tabular}

La dérivation sous (15) du pluriel en (14a) montre que le gabarit du pluriel est de $5 \mathrm{CV}$.

(15)

\begin{tabular}{|c|c|c|c|c|c|c|c|c|c|c|c|c|}
\hline \multirow[b]{2}{*}{ b. } & \multirow[b]{2}{*}{ /Sqiq/ } & \multicolumn{9}{|c|}{ gabarit pluriel : $5 \mathrm{CV}$} & \multicolumn{2}{|c|}{ suffixe } \\
\hline & & C & V & $=\mathrm{V}$ & C & $\mathrm{V}$ & C & $\mathrm{V}$ & C & V & C & V \\
\hline & & $\mid \delta$ & & $q$ & & & I & & $q$ & & $n$ & \\
\hline & & & & & & & & & & & & \\
\hline
\end{tabular}

La racine ${ }^{8}$ est associée à un gabarit de $5 \mathrm{CV}$. Comme elle est trilitère, $\mathrm{C} 2$ gémine pour occuper le gabarit de $5 \mathrm{CV}$. Quant au suffixe du pluriel externe, il a son CV lexical auquel il s'associe au moment de la dérivation. 
Pour le même pluriel, on a un singulier différent dans les 3 derniers exemples en (14a). La dérivation de ce type de singulier est donnée sous (16).

(16)

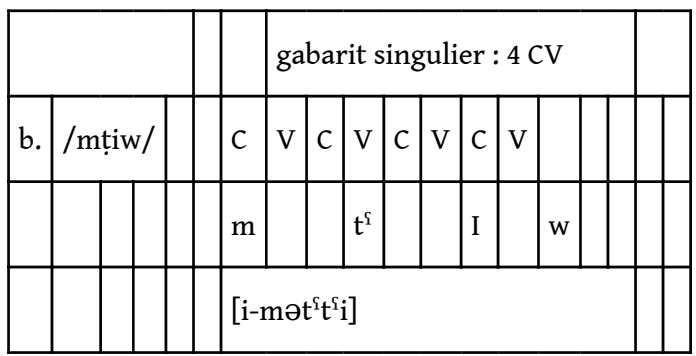

On voit que le $w$ final n'a pas de position consonantique pour s'associer au gabarit, ce qui fait qu'il reste flottant.

Le pluriel VCCVC-n en (14b) ne contient que des noms à voyelle initiale radicale. Les représentations lexicales sous (17) montreront la dérivation du pluriel sur un gabarit de $5 \mathrm{CV}^{9}$.

(17)

\begin{tabular}{|c|c|c|c|c|c|c|c|c|c|c|c|c|c|}
\hline & D & & & & arit & plu & iel : & $5 \mathrm{C}$ & & & & suf & fixe \\
\hline /anzað/ & $c$ & $\mathrm{v}$ & C & $\mathrm{v} \mid$ & & 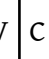 & $\mathrm{V}$ & C & & V & & C & $\mathrm{V}$ \\
\hline & & & & A & & $\mathrm{z}^{\mathrm{S}}$ & & A & co & & & $\mathrm{n}$ & \\
\hline & & & & $n z^{f} a^{2}$ & & & & & & & & & \\
\hline
\end{tabular}

Et voici sous (18) la dérivation du singulier associé.

(18)

\begin{tabular}{|c|c|c|c|c|c|c|c|c|c|c|c|c|c|}
\hline & & D & & & ar & it si & ngul & ier : & $5 \mathrm{Cl}$ & & & & \\
\hline b & /anzað/ & c & $\mathrm{v}$ & c & V & c & C & $\mathrm{v}$ & c & & 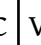 & & \\
\hline & & & & & $\mathrm{A}$ & $\mathrm{n}$ & $z^{\mathrm{q}}$ & & A & & & & \\
\hline & & & & & & & б] & & & & & & \\
\hline
\end{tabular}

La représentation du pluriel urgal-n est donnée sous (19) avec le singulier associé sous (20).

(19)

\begin{tabular}{|l|l|l|l|l|l|}
\hline & & D & & gabarit pluriel $: 5 \mathrm{CV}$ & suffixe \\
\hline
\end{tabular}




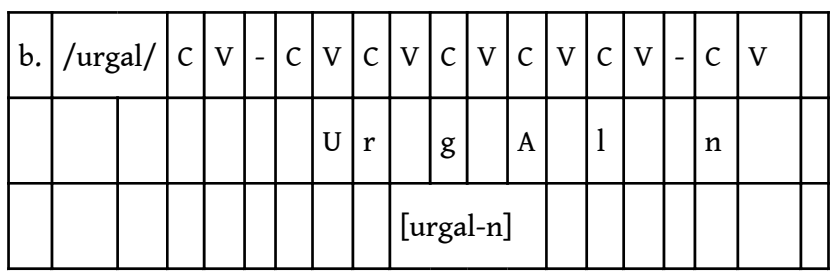

(20)

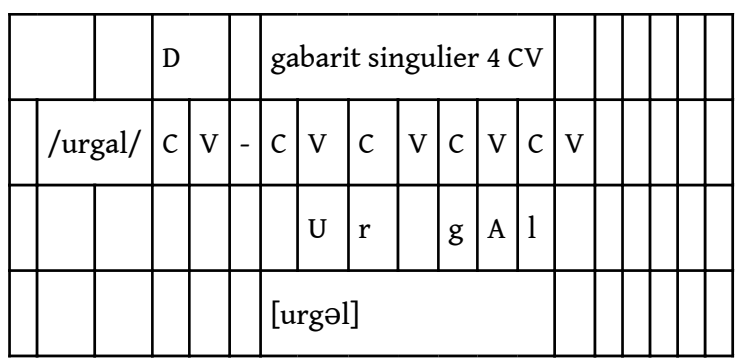

Le singulier est fait sur la même racine que le pluriel /urgal/ qui contient un A entre C2 et $\mathrm{C} 3$. Or son association au gabarit à $4 \mathrm{CV}$ ne laisse pas assez d'espace à tous les éléments pour s'exprimer. Ce qui fait que la voyelle $a$ en position V2 est associée à une seule position vocalique et se réalise schwa.

Les représentations sous (21) montrent la dérivation d'un des singuliers des deux derniers exemples illustrés en (14b).

(21)

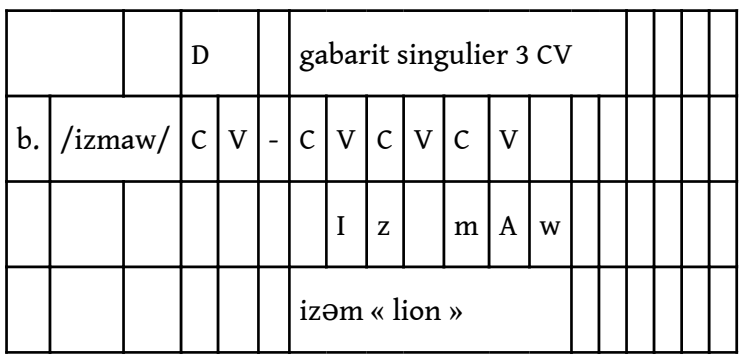

Pour ce type de singulier, comme on le voit sous (21), la racine est associée à un gabarit de $3 \mathrm{CV}$, ce qui fait que la consonne finale w demeure flottante (et donc non-réalisée), et que V2 est associée à une seule position vocalique et se réalise comme zéro en finale de $\operatorname{mot}^{10}$. Ce qui donne la forme de surface [izəm].

En revanche, au pluriel sous (22), comme le gabarit est de $5 \mathrm{CV}$, tous les éléments mélodiques de la racine sont associés et donc réalisés en surface.

(22)

\begin{tabular}{|c|c|c|c|c|c|c|c|c|c|c|c|c|c|c|c|}
\hline & & D & & & & ba & it & lu & el & 5 & & & & & \\
\hline b. & /IzmAw/ & c & V & C & V & C & $\mathrm{V}$ & $C$ & V & C & V & $c$ & V & C & V \\
\hline & & & & & I & $\mathrm{z}$ & & $\mathrm{m}$ & & A & & $\mathrm{W}$ & & $n$ & \\
\hline
\end{tabular}




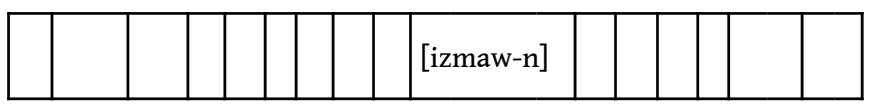

Le $3^{\text {ème }}$ type du pluriel du tableau (14c) sera analysé de la même manière. Sa représentation lexicale montre que son gabarit est fait de $5 \mathrm{CV}$.

(23)

\begin{tabular}{|l|l|l|l|l|l|l|l|l|l|l|l|l|}
\hline \multicolumn{7}{|c|}{ pluriel 5CV } & & \multicolumn{2}{|l|}{} & \multicolumn{2}{c|}{ suffixe } \\
\hline C & V & C & V & C & V & C & V & C & V & - & C & V \\
\hline z & & U & & l & & I & & B & & & n & \\
\hline i-zulis-n & & & & & & & \\
\hline
\end{tabular}

Les deux derniers pluriels sous (14c) ont la même représentation, à ceci près que la consonne C3 est absente au singulier. Comme auparavant, j'en conclus que cette consonne fait partie de la racine mais ne peut s'associer à son gabarit du singulier qui est trop petit. Voici sous (24) la représentation de ces pluriels ainsi que de leurs singuliers associés sous (25).

0

\begin{tabular}{|c|c|c|c|c|c|c|c|c|c|c|c|}
\hline & & & & gabar & it $p$ & uri & $\mathrm{el}:$ & & & & \\
\hline b. & /juðuj/ & c & 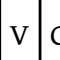 & V & C & & $C$ & c & V & d & $V$ \\
\hline & & $\mathrm{j}$ & L & & д & & U & $\mathrm{j}$ & & $\mathrm{n}$ & \\
\hline & & & uð̃uj & & & & & & & & \\
\hline
\end{tabular}

Au singulier sous (25), la même racine est associée à un gabarit de $4 \mathrm{CV}$, ce qui fait que la consonne $j$ en position $C 3$ reste flottante.

(25)

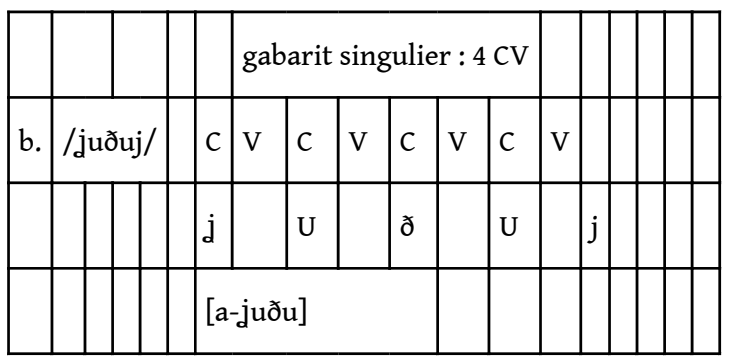

Pour conclure, dans la démonstration supra, j'ai tenté de montrer que certains éléments lexicaux des racines ne font pas surface au singulier parce que le gabarit de leur classe ne leur laisse pas suffisamment d'espace. Mon analyse montre que la formation du pluriel en kabyle des At Mangellat (Ain El Hammam) n'est pas aussi 
complexe et irrégulière que le présentent les grammaires traditionnelles. Il ressort de ce qui précède que le gabarit des différents pluriels est uniforme, i.e. fait de 5 unités CV. Contrairement au pluriel, il n'y a pas de restriction concernant la taille au singulier. C'est la raison pour laquelle la formation du pluriel semble montrer des distorsions par rapport au singulier :

Le gabarit nominal $:$ singulier = tailles différentes vs pluriel = une seule taille

Cette dissymétrie fait que des éléments mélodiques peuvent apparaître au pluriel mais sont absents au singulier. Enfin, le scénario diachronique que l'on peut déduire est que les noms analysés peuvent avoir été à l'origine associés au même squelette bien que certains aient perdu un morceau de leur structure : certains éléments mélodiques sont toujours présents dans le lexique mais ne peuvent pas faire surface.

\section{BIBLIOGRAPHIE}

ACHAB, K., " Alternation of State in Berber ", dans Research in Afroasiatic Grammar, Jacqueline Lecarme (dir(s).), Benjamins, Amsterdam, 2003, 1-20.

BASSET, A., « Note sur le genre et le nombre en berbère », in Cinquantenaire de la Faculté d'Alger, 1932, 62-71.

BASSET, A., « Sur la voyelle initiale en berbère. ", Revue Africaine 86, 1945, 82-88.

BASSET A., La langue berbère, Londres, 1952.

BENDJABALLAH, S., Aspect du système verbal berbère (kabyle). Mémoire de DEA, Université de Paris 7, 1995.

BENDJABALLAH, S., Trois figures de la structure interne des gabarits : activité morphologique du niveau squelettal des représentations phonologiques en berbère, somali et béja. Thèse de Doctorat, université de Paris 7, 1999.

BENDJABALLAH, S., « The negative preterite in Kabyle Berber », Folia Linguistica 34, 2001, 185-223.

BENDJABALLAH, S., « Longueur phonologique des voyelles en kabyle », Etudes et documents berbères 22, 2005, 47-69.

BENDJABALLAH, S., « Note sur la voyelle initiale stable en kabyle », dans Parcours berbères, Mélanges offerts à Paulette Galand-Pernet et Lionel Galand pour leur 90e anniversaire, Amina Mettouchi (dir(s).), Rüdiger Köppe Verlag, Köln, 2011, 417-434.

BENDJABALLAH, S. et M. HAIDEN, « A Typology of Emptiness in Templates », dans Sounds of Silence, Jutta Hartmann, Veronika Hegedus \& Henk C. van Riemsdijk (dir(s).), Hartmann, Amsterdam, 2008, 23-59.

BENDJABALLAH, S. et M. HAIDEN, « The representational anomalies of floating markers: light prepositions in Taqbaylit of Chemini ", dans Challenges to Linearization, Theresa Biberauer \& Ian Roberts (dir(s).), Mouton de Gruyter, Berlin, 2013, 331-375. 
BEN SI SAID, S., « Association sous contrôle grammatical : le cas du A du pluriel externe en kabyle », Acta Linguistica Hungarica 57, 2010, 432-443.

BEN SI SAID, S., « Interaction between structure and melody: the case of Kabyle nouns », dans On Words and Sounds: A Selection of Papers from the 40th PLM, Kamila DĘbowska-Kozłowska et Katarzyna Dziubalska-Kołaczyk (dir(s).), Cambridge Scholars Publishing, Newcastle, 2011, 39-48.

BEN SI SAID, S., De la nature de la variation diatopique en kabyle : étude de la formation des singulier et pluriel nominaux. Thèse de doctorat, Université de Nice Sophia Antipolis, 2014.

BEN SI SAID, S., « La voyelle initiale des noms et l'état d'annexion en kabyle (berbère) », Revue Canadienne de Linguistique, 65 (2), 2020, 155-180.

BOERSMA, P., Functional phonology: formalizing the interaction between articulatory and perceptual drives. Thèse de Doctorat, University of Amsterdam, 1998.

BRUGNATELLI, V., « Deux notes sur l'état d'annexion en berbère », in Proceeding of 4th international Hamito-Semitic Congress, Amsterdam, 1987, 349-359.

BRUGNATELLI, V., " L'état d'annexion en diachronie », in Afroasiatica Neapolitana. Contributi presentati all' $8^{\circ}$ Incontro di Linguistica Afroasiatica, TOSCO M. et BAUSI A. (dir(s).), Istituto Universitario Orientale, Napoli, 1997, 139-150.

BUCCI, J., « Voyelles longues virtuelles et réduction vocalique en coratin », Revue canadienne de linguistique, 58 (3), 2013, 397- 414.

CHAKER, S., Un parler berbère d'Algérie (syntaxe), Publications de l'Université de Provence, Aix-enProvence, 1983.

COHEN D., « Racines », dans A la croisée des études libyco-berbères. Mélanges offerts à Paulette Galand-Pernet et Lionel Galand, J. Drouin et A. Roth (eds.), Geuthner, Paris, 61-175, 1993.

DALLET, J-M., Dictionnaire kabyle-français, Selaf, Paris, 1982.

EL HAMDI, F., On Tachelhit root structure and its implication for the organization of the lexicon. Thèse de doctorat, université de Rabat et université Paris 8, 2018.

ENCREVE, P., La liaison avec et sans enchaînement : phonologie tridimensionnelle et usages du français, Seuil, Paris, 1988.

FLEMMING, E., « Contrast and perceptual distinctiveness », dans Phonetically-based Phonology, Bruce Hayes, Robert Kirchner et Donca Steriade (dir(s).), CUP, Cambridge, 2004, 232-276.

FODOR, J., The Modularity of the Mind, MIT-Bradford, Cambridge, 1983.

GOLDSMITH, J. A., Autosegmental Phonology. Thèse de doctorat, MIT, 1976.

GUERSSEL, M., « On the Case System of Berber », Revue Canadienne de Linguistique, 37 (2), 1992, 175-195.

HAMANN, S., « The Phonetics-Phonology Interface », dans Continuum Companion to Phonology, Nancy Kula, Bert Botma et Kuniya Nasukawa (dir(s).), London, 2011, 202-224.

HAMMOND, M., « Vowel Quantity and Syllabification in English », Language 73, 1997, 1-17.

HARRIS, J., « Phonological representations are redundancy-free and fully interpretable », dans Current trends in phonology: models and methods, Jacques Durant et Bernard Laks (dir(s).), European Studies Research Institute, Manchester, 1996, 305-332.

HARRIS, J. et G. LINDSEY, « The elements of phonological representation », dans Frontiers of Phonology: atoms, structures, derivations, Francis Katamba (dir(s).), Longman, Essex, 
1995, 34-79.

HYMAN, L., « Tone: Is it different? » In The Handbook of Phonological Theory," 2nd edition, John Goldsmith, Jason Riggle and Alan Yu (dir(s).), Blackwell, 2011, 197-239.

IDRISSI, A., Towards a Root-and-Template Approach to Shape-Invariant Morphology.

Thèse de doctorat, université de Québec à Montréal, 2000.

KAYE, J., « Government in phonology: the case of Moroccan Arabic », The Linguistic Review 6, 1990, 131-159.

KAYE, J. et J. LOWENSTAMM, « De la syllabicité », dans Forme sonore du langage, structure des représentations en phonologie, François Dell et Jean-Roger Vergnaud (dir)s.), Herman, Paris, 1984, 123-159.

KAYE, J., J. LOWENSTAMM et J.-R. VERGNAUD, « The internal structure of phonological representations: a theory of Charm and government », Phonology Yearbook 2, 1985, 305- 328.

KAYE, J., J. LOWENSTAMM et J.-R. VERGNAUD, « Constituent Structure and Government in Phonology ", Phonology 7(2), 1990, 193-231.

KINGSTON, J., «The phonetics-phonology interface », dans The cambridge Handbook of Phonology, Paul de Lacy (dir(s).), CUP, Cambridge, 2007, 401-434.

LAHROUCHI, M., Aspect morpho-phonologique de la dérivation verbale en berbère (tachelhit d'Agadir), Thèse de doctorat, Université de Paris 7, 2001.

LAHROUCHI, M., « Manifestations gabaritiques dans la dérivation verbale en berbère tachelhit », Recherches Linguistiques de Vincennes 32, 2003, 61-81.

LAHROUCHI, M., « A Templatic Approach to Gemination in the Imperfective Stem of Tashlhiyt Berber », Studies in African Linguistics 37, 2009, 21-60.

LAHROUCHI, M., « Templates, markers and syntactic structure in Tashlhiyt Berber », Lingua 133, 2013, 53-72.

LEBEN, W., Suprasegmental phonology. Thèse de doctorat, MIT, 1973.

LOWENSTAMM, J., " Vocalic length and syllable structure in Semitic », dans Semitic Studies in Honor of Wolf Leslau on the Occasion of his 85th Birthday, Alan S. Kaye (dir), Harrassowitz Verlag, Wiesbaden, 1991, 949-965..

LOWENSTAMM, J., «CV as the only syllable type », dans Current trends in Phonology: Models and methods, Bernard Laks et Jacques Durand (dir)s.), Salford, Manchester, 1996, 419-441.

MAMMERI, M., Précis de grammaire berbère (kabyle), Awal, Paris, 1986.

McCARTHY, J. J., Formal problems in Semitic phonology and morphology. Thèse de doctorat, MIT, 1979.

McCARTHY, J. J., « A prosodic theory of nonconcatenative morphology », Linguistic Inquiry 12, $1981,373-418$.

McCARTHY, J. J., « Prosodic Template, Morphemic Template and Morphemic Tiers » dans The structure of phonological representations (Part I), Harry. van der Hulst. et Norval Smith (dir)s.), Foris, Dordrecht, 1982, 191-223.

McCARTHY, J. J., « Consonantal Morphology in the Chaha Verb », in Proceeding of the West coast conference on formal linguistics, Stanford, 1983, 176-188. 
McCARTHY, J. J. et A. S. PRINCE, « Prosodic Morphology », dans Phonological theory: the essential reading, John. Goldsmith (dir(s).), Oxford, 1999, 238-288.

PASTER, M., « Floating tones in Gã », Studies in African Linguistics 32(1), 2003, 17-39.

SCHEER, T., A Lateral Theory of Phonology vol. 1: What is CVCV, and why should it be?, Mouton de Gruyter, Berlin, 2004.

SCHEER T., « Spell-out, post-phonological » dans Crossing Phonetics-Phonology Lines, Eugeniusz Cyran et Jolanta Szpyra-Kozłowska (dir(s).), Cambridge Scholars, Newcastle, 2014, 255-275.

STUMME, H., Handbuch des Schilhischen von Tazerwalt, Hinrichs, Leipzig, 1899.

VYCICHL, W., «L'article défini du berbère », Dans Mémorial André Basset, Maisonneuve, Paris, 1957, 139-146.

WOOHYEOK, C., « On the Relation between Phonetics and Phonology », Linguistic Research 29(1), 2012, 127-156.

ZIKOVA, M. et T. SCHEER, « The Havlík Pattern and Directional Lower », dans Formal Approach to Slavic Linguistics, D. Zec et W. Browne (dir)s.), Cornell University, 2009, 471-486.

ZIMMERMANN, E., « An argument for subtonal features : Floating tones in two Otomanguean languages », Acta Linguistica Academica 64, 2017, 619-656.

\section{NOTES}

1. Les données du kabyle analysées ici sont issues du dictionnaire kabyle-français de Dallet (1982) que j'ai transformé en base de données interrogeable sous Access. Je précise que la variété du kabyle en question est parlée à At Mangellat (Ain El Hammam), dont je suis un locuteur natif.

2. Dans la présentation des données de cet article, la voyelle initiale dite «instable» (diachroniquement préfixale) est décalée du radical, car elle n'appartient pas à la racine contrairement à la voyelle initiale dite « stable » ou « constante ». C'est l'état d'annexion qui peut nous informer du statut de la voyelle initiale des noms, si elle est radicale ou pas: la voyelle

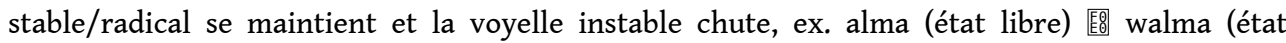
d'annexion) a-rgaz (état libre) 㕌 w-rgaz (état d'annexion). Pour plus de détail sur la question, voir (Basset 1945, Bendjaballah 2011, Ben Si Said 2020, 2014 et Idrissi 2000 entre autres).

3. La forme [if]] est aussi attestée au singulier dans certaines variétés du kabyle mais le pluriel demeure le même [afSiw-n]. Ben Si Said (2014) donne une analyse détaillée de la variation diatopique des singuliers et pluriels nominaux en kabyle, où il fait une généralisation du gabarit pluriel à $5 \mathrm{CV}$ sur l'ensemble de la diatopie.

4. L'évolution du model CVCV depuis la phonologie autosegmental des années 70 est retracée en détail dans Scheer (2004).

5. Je précise qu'une attaque branchante est toujours un groupe $T R(T=0 b s t r u a n t e / R=$ sonante) mais que l'inverse n'est pas vrai. Il existe des TR qui sont associés à une seule position syllabique, instanciant une structure de contour (au même titre que les affriquées). Entre les membres d'une attaque branchante (bipositionnelle) il existe une relation latérale au niveau de leur contenu mélodique : le GI (Gouvernement Infrasegmental, Scheer 2004 : § 14).

6. Il y a aussi des approches qui considèrent que la phonétique et la phonologie ont un seul système computationnel (cf. Flemming 2004).

7. Je précise que seule la voyelle initiale radicale apparait dans les représentations. Les détails sur la motivation de ces représentations sont donnés dans Bendjaballah (2011) et Ben Si Saïd (2014, 2020). 
8. L'analyse implique l'idée que la racine est vocalisée, c'est-à-dire qu'elle contient des voyelles (Basset 1952, Cohen 1993 et El Hamdi 2018 pour une analyse plus récente et détaillée).

9. La voyelle initiale radicale (dite stable) s'associe sur la première position $\mathrm{V}$ de la racine ou du gabarit et la position V du déterminant D (cf. Guerssel 1992). Le gabarit lexical des radicaux à voyelle initiale stable est "infirme»; c'est au cours de la dérivation du nom que la voyelle initiale du radical accède à une seconde position $\mathrm{V}$, et peut être réalisée phonétiquement. Pour plus de détail sur la motivation de la représentation phonologique de la voyelle initiale sous (16) à (19), voir Bendjaballah (2011) et Ben Si Said (2020) et pour la représentation syntaxique du déterminant (D), voir Achab (2003) et Guerssel (1992), entre autres.

10. Le schwa en finale de mot (ə\#) est absent dans la phonologie du kabyle et donc, même s'il existe dans la forme sous-jacente, il n'est pas réalisé en surface.

\section{RÉSUMÉS}

Le fait que des objets phonologiques puissent être présents dans l'entrée lexicale de la racine mais absents de la réalisation de surface est rendu possible par la configuration restreinte de l'espace syllabique : la structure n'offre pas assez d'espace à tous les éléments mélodiques pour s'associer et se réaliser au niveau de la surface. Le pluriel externe kabyle présente des éléments mélodiques qui ne sont pas présents au singulier, par exemple iddəw iddaw-n "singe", iccəw acciw- $n$ "corne", izəm izmaw- $n$ "lion" (-n est le suffixe du pluriel). Comme ces éléments ne peuvent être prédits, ils doivent appartenir à la racine et ils sont absents au singulier en raison de la restriction de l'espace syllabique, le gabarit. Ma proposition est que la forme du singulier dans les noms kabyles peut être contrainte par la taille du gabarit, c'est-à-dire qu'en kabyle l'interprétation de la mélodie est contrainte par la structure syllabique.

The fact that phonological objects may be present in the lexical entry of the root but absent from the surface realization is made possible by the restricted configuration of the syllabic space: the structure does not offer enough space for all the melodic elements to be associated and realized at the surface. The external plural in Kabyle presents melodic elements that are absent in the singular form, e.g. iddəw iddaw-n "monkey", iccəw acciw-n "horn", izəm izmaw- $n$ "lion" (-n is the suffix of the plural). Since these elements cannot be predicted, they must belong to the root and they are absent in the singular because of the restriction of the syllabic space, the template. My proposal is that the form of the singular in Kabyle nouns can be constrained by the size of the template, i.e. in Kabyle the interpretation of the melody is constrained by the syllabic structure.

\section{INDEX}

Mots-clés : langue kabyle, gabarit, structure syllabique, pluriel, singulier

Keywords : kabyle language, template, syllabic structure, plural, singular 


\section{AUTEUR}

\section{SAMIR BEN SI SAID}

Centre de Recherche en Langue et Culture Amazighes (CRLCA), Bejaia, Algérie 